\title{
Laser spectroscopy of NiBr: New electronic states and hyperfine structure
}

\author{
Jianjun Ye, J. W.-H. Leung, and A. S.-C. Cheung ${ }^{\text {a) }}$ \\ Department of Chemistry, The University of Hong Kong, Pokfulam Road, Hong Kong
}

(Received 21 August 2006; accepted 3 November 2006; published online 6 December 2006)

\begin{abstract}
Laser induced fluorescence spectrum of $\mathrm{NiBr}$ in the visible region between 604 and $666 \mathrm{~nm}$ has been recorded and analyzed. Fourteen bands belonging to three electronic transition systems, namely, [15.1 $]^{2} \Delta_{5 / 2}-X^{2} \Pi_{3 / 2},[15.1]^{2} \Pi_{3 / 2}-X^{2} \Pi_{3 / 2}$, and [14.0 $]^{2} \Delta_{5 / 2}-X^{2} \Pi_{3 / 2}$ have been observed. Spectra of isotopic molecules were also observed and analyzed. Detailed analysis of the recorded spectra indicated that the two electronic states $[15.1]^{2} \Pi_{3 / 2}$ and $[15.1]^{2} \Delta_{5 / 2}$ lie about $1 \mathrm{~cm}^{-1}$ apart from each other and $J$-dependent perturbation due to spin-uncoupling interaction has been observed. Least squares fitting procedures involving deperturbation matrix elements were used to fit the observed line positions, which yielded accurate molecular constants for the $[15.1]^{2} \Pi_{3 / 2}$ and $[15.1]^{2} \Delta_{5 / 2}$ states. In addition, the $(1,0)$ band of the $[15.1]^{2} \Delta_{5 / 2}-X^{2} \Pi_{3 / 2}$ transition shows partially resolved hyperfine structure that was caused by the interaction of unpaired electron with the magnetic moment of the Br nucleus (nuclear spin of $I=3 / 2$ ) in the excited state. The rapid decrease in hyperfine width as $J$ increases suggests that the hyperfine coupling in the excited state conforms to Hund's case $\left(\mathrm{a}_{\beta}\right)$ coupling scheme. (C) 2006 American Institute of Physics.

[DOI: $10.1063 / 1.2402170]$
\end{abstract}

\section{INTRODUCTION}

Considerable efforts have recently been devoted to the studies of transition metal compounds to understand their spectroscopic properties. ${ }^{1,2}$ These studies begin with diatomic molecules involving one transition metal element and one main group element from group IV to VII in the periodic table, where major interest lies in the understanding of the chemical bonding involved and the determination of molecular properties. Such diatomic molecules can be viewed as the transition metal atom is subjected to the influence of a ligand (main group atom). Besides oxides, ${ }^{3}$ carbides and nitrides ${ }^{4}$ and recently monohalides ${ }^{2,5}$ have been reported. They are the simplest examples for studying metal-ligand interaction. Spectroscopic investigation of the diatomic compounds yields molecular properties such as bond length and bond strength and also gives information concerning electronic structure and the occupation of molecular orbitals formed from a transition metal atom and a main group element. Despite of the fact that these are diatomic molecules, the electronic transition spectrum is often quite complex and congested. This is because the fivefold degenerate $d$ orbitals of the transition metal atom make possible electronic states that are close in energy and the unpaired electrons will give rise to many close-lying electronic states with high spin multiplicity. In addition, transition metal and main group atoms often have isotopes with appreciable abundance and the isotope spectra cause serious spectral overlap in the near vicinity. Furthermore, perturbations and hyperfine structure are frequently found in the spectrum of these molecules. Highresolution spectroscopic techniques are proven to be necessary for detailed studies of these molecules.

\footnotetext{
${ }^{a)}$ Author to whom correspondence should be addressed. Fax: 28571586 . Electronic mail: hrsccsc@hku.hk
}

Amongst the nickel monohalide diatomic compounds, much high-resolution work was performed to NiF (Refs. 6-17) and $\mathrm{NiCl}$ (Refs. 18-24) earlier and good knowledge of the electronic structure of these nickel monohalides has been obtained. However, our understanding of NiBr (Refs. 25-31) and NiI (Refs. 32-36) is still quite fragmented. We have performed high-resolution spectroscopic studies to these molecules and the amount of spectroscopic information obtained for the excited states for $\mathrm{NiBr}$ and $\mathrm{NiI}$ is still quite limited when compared with the other two nickel monohalides. Electronic emission transition bands of $\mathrm{NiBr}$ in the UV and visible regions between 3800 and $5000 \AA$ were recorded. Only vibrational constants were reported using the conventional grating spectroscopy. Recently, Leung et al. ${ }^{30}$ using laser vaporization/reaction free jet expansion and laser induced fluorescence spectroscopy identified and confirmed that the $X^{2} \Pi_{3 / 2}$ state is the ground state and the next lowlying state is $A^{2} \Delta_{5 / 2}$ state, and determined that the two states are only $37.25 \mathrm{~cm}^{-1}$ apart. Yamazaki et al. ${ }^{31}$ using millimeter wave spectroscopy studied the perturbation of these two states at high $J$ values. Two more electronic states, namely, $[13.2]^{2} \Pi_{3 / 2}$ and $[12.6]^{2} \Sigma^{+}$, have also been identified and characterized. Both vibrational and rotational constants for all four electronic states were reported.

In this paper, we describe the analysis of 14 electronic transition bands, belonging to three new electronic systems, recorded using the technique of laser vaporization/reaction free jet expansion and laser induced fluorescence spectroscopy. The three electronic transitions identified are $[15.1]^{2} \Delta_{5 / 2}-X^{2} \Pi_{3 / 2}, \quad[15.1]^{2} \Pi_{3 / 2}-X^{2} \Pi_{3 / 2}, \quad$ and $[14.0]^{2} \Delta_{5 / 2}-X^{2} \Pi_{3 / 2} \quad$ systems. The $[15.1]^{2} \Delta_{5 / 2}$ and $[15.1]^{2} \Pi_{3 / 2}$ states are only $1 \mathrm{~cm}^{-1}$ apart; perturbations between the different vibronic states were observed and satisfactorily explained. In addition, hyperfine interaction arising 


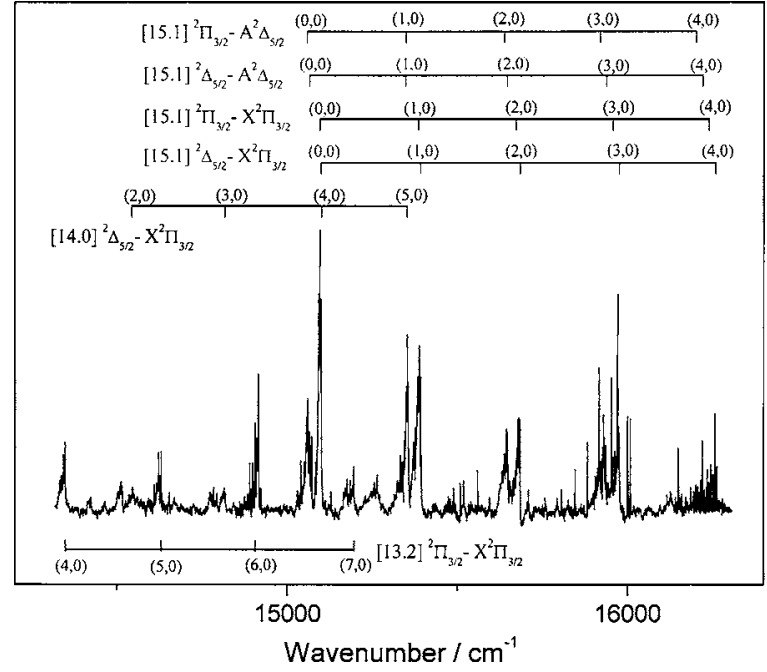

FIG. 1. Broadband low-resolution LIF spectrum of the $[15.1]^{2} \Delta_{5 / 2}-X^{2} \Pi_{3 / 2}$, $[15.1]^{2} \Delta_{5 / 2}-A^{2} \Delta_{5 / 2}, \quad[15.1]^{2} \Pi_{3 / 2}-X^{2} \Pi_{3 / 2}, \quad[15.1]^{2} \Pi_{3 / 2}-A^{2} \Delta_{5 / 2}$, $[14.0]^{2} \Delta_{5 / 2}-X^{2} \Pi_{3 / 2}$, and [13.2 ${ }^{2} \Pi_{3 / 2}-X^{2} \Pi_{3 / 2}$ band systems of the $\mathrm{NiBr}$.

from unpaired electrons and the magnetic moment of the bromine atom with a nuclear spin of $I=3 / 2$ has also been observed and analyzed.

\section{EXPERIMENT}

The experimental apparatus and detailed procedure for producing transition metal containing molecules using laser vaporization/reaction free jet expansion and LIF spectroscopy have been described in earlier publication. ${ }^{34}$ Only a brief description of the relevant experimental conditions for obtaining the $\mathrm{NiBr}$ spectrum is given here. Pulses of laser radiation at $532 \mathrm{~nm}, 9-10 \mathrm{~mJ}$, and $10 \mathrm{~ns}$ from a Nd:YAG (yttrium aluminum garnet) laser were focused onto the surface of a nickel rod to generate nickel atoms. A pulsed valve, synchronized with appropriate delay time, released gas mixture of $2 \% \mathrm{C}_{2} \mathrm{H}_{5} \mathrm{Br}$ in argon to react with the nickel atom to produce NiBr. The Nd:YAG-pulsed valve system was operated at $10 \mathrm{~Hz}$. Argon ion laser pumped cw ring dye laser operating with DCM and R6G (Exciton, Inc.) dyes in the visible region and argon ion laser pumped cw ring Ti:sapphire laser in the near infrared region were used to excite the jet cooled $\mathrm{NiBr}$ molecules. LIF signal was collected by a lens system and detected by a photomultiplier tube. A linewidth of about $250 \mathrm{MHz}$ was obtained. The wavelength of the dye laser was measured using a wavemeter with an accuracy of one part in $10^{7}$. The absolute accuracy of the measured line position is about $\pm 0.002 \mathrm{~cm}^{-1}$.

\section{RESULTS AND DISCUSSION}

Figure 1 shows a broadband low-resolution scan of the $\mathrm{NiBr}$ spectrum in the visible region between 14300 and $16600 \mathrm{~cm}^{-1}$. From the low-resolution LIF spectrum, the four strong bands near $15100 \mathrm{~cm}^{-1}$ are the $(0,0)$ bands of the $[15.1]^{2} \Pi_{3 / 2}-X^{2} \Pi_{3 / 2},[15.1]^{2} \Delta_{5 / 2}-X^{2} \Pi_{3 / 2}$, $[15.1]^{2} \Pi_{3 / 2}-A^{2} \Delta_{5 / 2}$, and $[15.1]^{2} \Delta_{5 / 2}-A^{2} \Delta_{5 / 2}$ systems. Vibronic transition bands $\left(v^{\prime}, 0\right)$ with $v^{\prime}=0-5$ to of these four

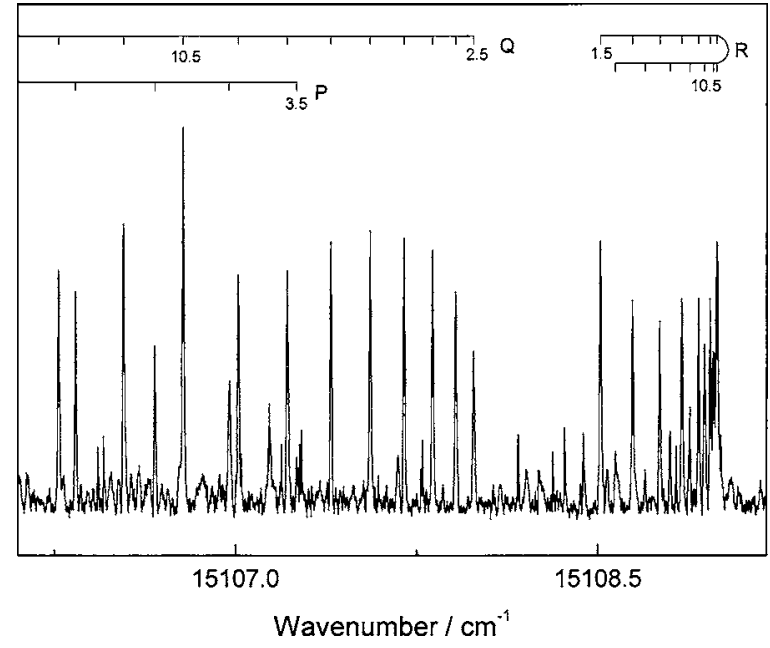

FIG. 2. $(4,0)$ band of the $[14.0]^{2} \Delta_{5 / 2}-X^{2} \Pi_{3 / 2}$ transition of ${ }^{58} \mathrm{Ni}{ }^{79} \mathrm{Br}$.

systems have been recorded and analyzed. Another system, the $[14.0]^{2} \Delta_{5 / 2}-X^{2} \Pi_{3 / 2}$ transition, with vibronic bands $(0,0),(4,0)$, and $(5,0)$ was also recorded and analyzed.

\section{A. $[14.0]^{2} \Delta_{5 / 2}-X^{2} \Pi_{3 / 2}$ system}

Two band heads were located at 15108 and $15380 \mathrm{~cm}^{-1}$, which were assigned as the $(4,0)$ and $(5,0)$ bands. Due to limitation in our dye laser wavelength coverage, molecular transition $\left(v^{\prime}, 0\right)$ bands with $v^{\prime}=1-3$ were not recorded. The $(0,0)$ band head was observed at $13986 \mathrm{~cm}^{-1}$ using Ti:sapphire laser with short wave (SW) optics. The vibrational quantum number assignment was confirmed by examining the isotopic shift between ${ }^{58} \mathrm{Ni}{ }^{81} \mathrm{Br}$ and ${ }^{58} \mathrm{Ni}{ }^{79} \mathrm{Br}$ molecules. Each of these bands consists of resolved $P, Q$, and $R$ branches. Line assignment was simple because the first line of each branch was observed. Figure 2 shows the band head region of the $(4,0)$ band. The observation of $P(3.5), Q(2.5)$, and $R(1.5)$ lines establishes that this is a $\Omega^{\prime}=2.5$ and $\Omega^{\prime \prime}=1.5$ transition. Furthermore, the $Q$ and $R$ branches are stronger than the $P$ branch, which is consistent with a $\Delta \Lambda=+1$ transition. Since the spectrum was recorded at relatively low temperature, only low $J$ lines $(J \leqslant 20.5)$ were observed and no $\Lambda$-type doubling was detected. The observed line positions were fit to a standard formula ${ }^{37}$

$$
\begin{aligned}
\nu_{0}= & T_{0}+B^{\prime} J^{\prime}\left(J^{\prime}+1\right)-D^{\prime}\left[J^{\prime}\left(J^{\prime}+1\right)\right]^{2} \\
& -\left\{B^{\prime \prime} J^{\prime \prime}\left(J^{\prime \prime}+1\right)-D^{\prime \prime}\left[J^{\prime \prime}\left(J^{\prime \prime}+1\right)\right]^{2}\right\},
\end{aligned}
$$

where the ' and " refer to the upper and the lower states, respectively. The ground state molecular constants were fixed to those obtained by Yamazaki et al.. ${ }^{31}$ Isotopic molecular transition lines of ${ }^{58} \mathrm{Ni}{ }^{79} \mathrm{Br},{ }^{58} \mathrm{Ni}{ }^{81} \mathrm{Br}$, and ${ }^{60} \mathrm{Ni}{ }^{79} \mathrm{Br}$ were measured and fitted. Molecular constants obtained are listed in Table I. A list of the measured line positions of the observed transition bands of the $[14.0]^{2} \Delta_{5 / 2}-X^{2} \Pi_{3 / 2}$ system of ${ }^{58} \mathrm{Ni}{ }^{79} \mathrm{Br},{ }^{58} \mathrm{Ni}{ }^{81} \mathrm{Br}$, and ${ }^{60} \mathrm{Ni}{ }^{79} \mathrm{Br}$ is available from the EPAPS. ${ }^{38}$ 
TABLE I. Molecular constants for the $[14.0]^{2} \Delta_{5 / 2}$ state of $\mathrm{NiBr}\left(\mathrm{cm}^{-1}\right)$.

\begin{tabular}{ccccc}
\hline \hline State & Parameter & ${ }^{58} \mathrm{Ni}{ }^{79} \mathrm{Br}$ & ${ }^{58} \mathrm{Ni}^{81} \mathrm{Br}$ & ${ }^{60} \mathrm{Ni}^{79} \mathrm{Br}$ \\
\hline$[14.0]^{2} \Delta_{5 / 2}$ & $T_{0}$ & 13986.150 & 13986.244 & $\ldots$ \\
& $B_{0}$ & 0.095221 & 0.094222 & \\
& $T_{4}$ & 15108.083 & 15102.618 & $\ldots$ \\
& $B_{4}$ & 0.093828 & 0.093237 & \\
& $T_{5}$ & 15380.738 & 15374.619 & 15396.061 \\
& $B_{5}$ & 0.093102 & 0.092496 & 0.091650 \\
\hline \hline
\end{tabular}

\section{B. $[15.1]^{2} \Delta_{5 / 2}-X^{2} \Pi_{3 / 2}$ system}

The $\left(v^{\prime}, 0\right)$ bands with $v^{\prime}=0-5$ have band heads observed at $15095,15390,15682,15973,16262$, and $16549 \mathrm{~cm}^{-1}$, respectively. The $R$ and $Q$ branches are stronger than the $P$ branch, which agrees well with a $\Delta \Lambda=+1$ transition. The first line of the $P, Q$, and $R$ branches are, respectively, with $J=3.5,2.5$, and 1.5 , which confirmed that the $\Omega^{\prime}=2.5$ and $\Omega^{\prime \prime}=1.5$. Figure 3 shows the band head region of the $(2,0)$ band. A list of the measured line positions of the observed transition bands of the $[15.1]^{2} \Delta_{5 / 2}-X^{2} \Pi_{3 / 2}$ system of ${ }^{58} \mathrm{Ni}{ }^{79} \mathrm{Br},{ }^{58} \mathrm{Ni}{ }^{81} \mathrm{Br},{ }^{60} \mathrm{Ni}{ }^{79} \mathrm{Br}$, and ${ }^{60} \mathrm{Ni}{ }^{81} \mathrm{Br}$ is available from the EPAPS. ${ }^{38}$

\section{C. $[15.1]^{2} \Pi_{3 / 2}-X^{2} \Pi_{3 / 2}$ system}

Similar to the $[15.1]^{2} \Delta_{5 / 2}-X^{2} \Pi_{3 / 2}$ system, the $\left(v^{\prime}, 0\right)$ bands with $v^{\prime}=0-5$ of the $[15.1]^{2} \Pi_{3 / 2}-X^{2} \Pi_{3 / 2}$ system have been observed. The band head positions of these bands are located at $15093,15384,15671,15956,16239$, and $16520 \mathrm{~cm}^{-1}$. Figure 4 shows the band head region of the $(2,0)$ band of the ${ }^{58} \mathrm{Ni}{ }^{79} \mathrm{Br}$ isotope. It can be seen easily that the $P$ and $R$ branches are stronger than the $Q$ branch, which agrees well with a $\Delta \Lambda=0$ transition. Furthermore, the first line of the $P, Q$, and $R$ branches are, respectively, with $J$ $=2.5,1.5$, and 1.5, which confirmed the assignment of the $\Omega^{\prime}=\Omega^{\prime \prime}=1.5$ value for both states. A list of the measured line positions of the observed transition bands of the $[15.1]{ }^{2} \Pi_{3 / 2}-X^{2} \Pi_{3 / 2}$ system of ${ }^{58} \mathrm{Ni}{ }^{79} \mathrm{Br}$ and ${ }^{58} \mathrm{Ni}^{81} \mathrm{Br}$ is available from the EPAPS. ${ }^{38}$

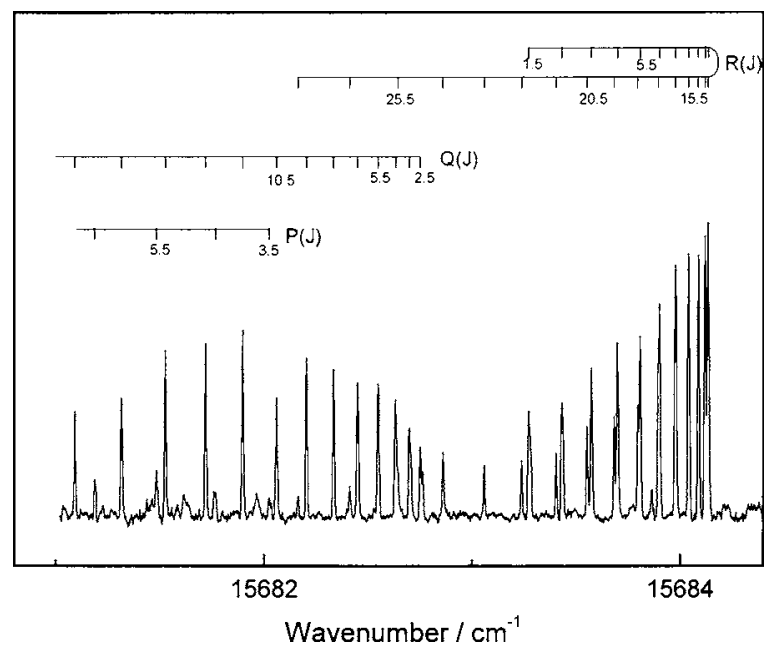

FIG. 3. $(2,0)$ band of the $[15.1]^{2} \Delta_{5 / 2}-X^{2} \Pi_{3 / 2}$ transition of ${ }^{58} \mathrm{Ni}{ }^{79} \mathrm{Br}$.

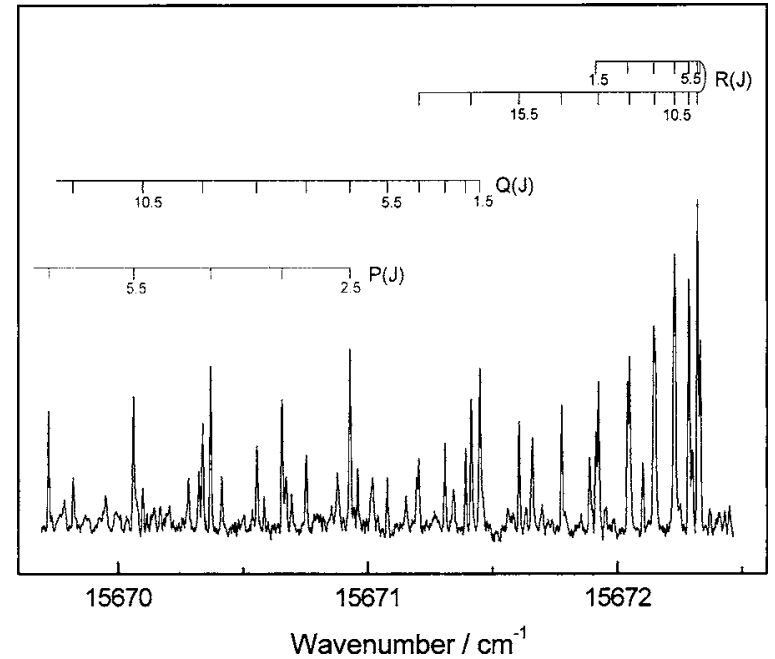

FIG. 4. (2,0) band of the $[15.1]^{2} \Pi_{3 / 2}-X^{2} \Pi_{3 / 2}$ transition of ${ }^{58} \mathrm{Ni}{ }^{79} \mathrm{Br}$.

\section{Interaction between the $[15.1]^{2} \Delta_{5 / 2}$ and the $[15.1]^{2} \Pi_{3 / 2}$ states}

Our analysis of the $[15.1]^{2} \Delta_{5 / 2}-X^{2} \Pi_{3 / 2}$ and $[15.1]^{2} \Pi_{3 / 2}-X^{2} \Pi_{3 / 2}$ transitions began with the $(4,0)$ and $(5,0)$ bands and the least squares fit was performed to individual band. These bands were the easiest ones to be analyzed because the spectra of the isotopic molecules are widespread and do not overlap with each other. Using standard parameters such as the band origin, $T_{0}$, and rotational constants $B$ and $D$ for the upper states and the known molecular constants for the ground state, the $(5,0)$ and $(4,0)$ bands were fit properly with the overall root mean square (rms) errors approaching the experimental limit of $0.002 \mathrm{~cm}^{-1}$. However, problems started to show up when we tried to fit the $(3,0)$, $(2,0)(1,0)$, and $(0,0)$ bands individually, and the $(0,0)$ band was the worst, for both the $[15.1]^{2} \Delta_{5 / 2}-X^{2} \Pi_{3 / 2}$ and $[15.1]^{2} \Pi_{3 / 2}-X^{2} \Pi_{3 / 2}$ transition systems. Individual bands could not be fit properly with this simple model because the energy level positions are shifted by an interaction, which has not been taken into account. Figure 5 depicts the term value plot of the $v=0-3$ levels of these two states, which

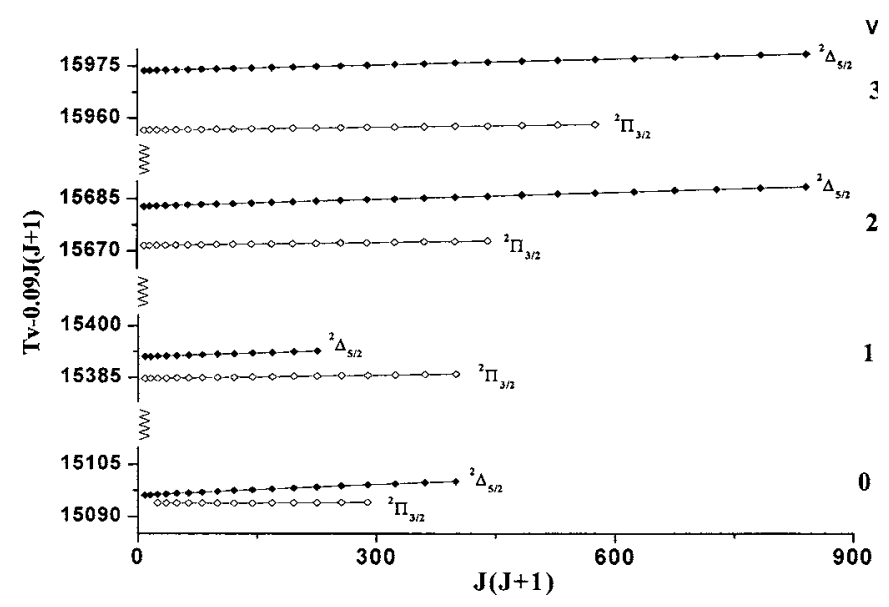

FIG. 5. Reduced term energy plot of the $v=0-3$ levels for the $[15.1]^{2} \Delta_{5 / 2}$ and $[15.1]^{2} \Pi_{3 / 2}$ states, showing an increase in separations between the vibronic levels as $v$ increases. 


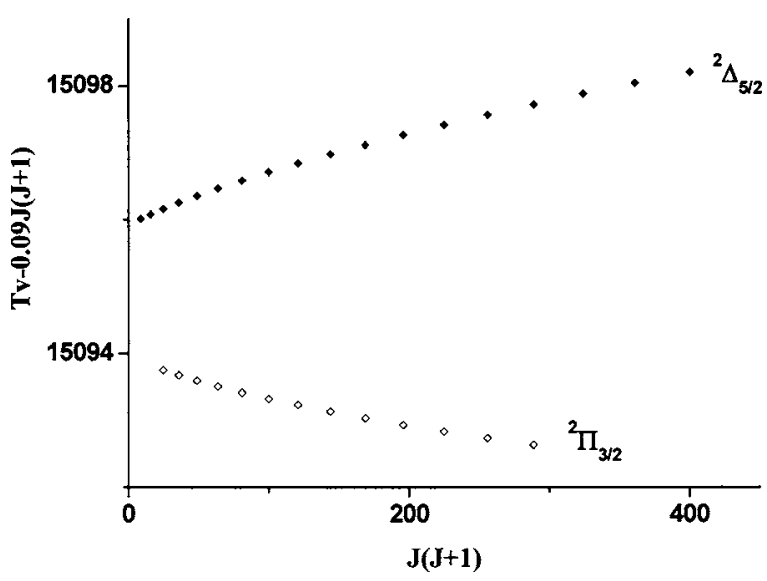

FIG. 6. Reduced term energy plot of the $v=0$ level of the $[15.1]^{2} \Delta_{5 / 2}$ and $[15.1]{ }^{2} \Pi_{3 / 2}$ states of ${ }^{58} \mathrm{Ni}{ }^{79} \mathrm{Br}$ plotted against $J(J+1)$. The slight curvature indicates the interaction between the two states.

shows that the $[15.1]^{2} \Delta_{5 / 2}$ and $[15.1]^{2} \Pi_{3 / 2}$ states are lying closer and closer as the vibrational quantum number decreases and the two vibronic levels of the states are interacting with each other. For the $v=3$ level, the separation between the two states is $17.6 \mathrm{~cm}^{-1}$, and the separation between the $v=0$ level, in fact, decreases to only $1.5 \mathrm{~cm}^{-1}$. Figure 6 shows a plot of the term values of the $v=0$ level on a reduced energy scale for both $[15.1]^{2} \Delta_{5 / 2}$ and $[15.1]^{2} \Pi_{3 / 2}$ states against $J(J+1)$. The curvature observed in the term value plot clearly indicates interactions between the $[15.1]^{2} \Delta_{5 / 2}$ and $[15.1]^{2} \Pi_{3 / 2}$ states.

The interaction between a ${ }^{2} \Pi$ state and a ${ }^{2} \Delta$ state was studied by Rice $e t$ al. ${ }^{39}$ The effective Hamiltonian and matrix elements for such interaction were worked out in detail. In order to fully describe the interactions between the ${ }^{2} \Pi$ and ${ }^{2} \Delta$ states, a $4 \times 4$ matrix would be necessary. Since the spinorbit interaction in $\mathrm{NiBr}$ is generally very large, the two spinorbit components of a doublet state is quite far apart and practically the observed spin components has been classified by the $\Omega$ value. This is the situation for the two interacting excited states that only one spin component of the doublet states $\left({ }^{2} \Delta\right.$ and ${ }^{2} \Pi$ states $)$, namely, $[15.1]^{2} \Delta_{5 / 2}$ and $[15.1]^{2} \Pi_{3 / 2}$ states, are observed. Under such circumstance, a $2 \times 2$ matrix is sufficient to account for the interaction and the following are the matrix elements in Hund's coupling case (a) scheme, ${ }^{39}$

$$
\begin{aligned}
\left\langle{ }^{2} \Pi_{3 / 2}|\hat{H}|^{2} \Pi_{3 / 2}\right\rangle= & T_{\Pi}+B_{\Pi}[(J-1 / 2)(J+3 / 2)-1] \\
& -D_{\Pi}\left\{[(J-1 / 2)(J+3 / 2)-1]^{2}\right. \\
& +(J-1 / 2)(J+3 / 2)]\}, \\
\left\langle{ }^{2} \Delta_{5 / 2}|\hat{H}|^{2} \Delta_{5 / 2}\right\rangle= & T_{\Delta}++B_{\Delta}[(J-3 / 2)(J+5 / 2)-2] \\
& -D_{\Delta}\left\{[(J-3 / 2)(J+5 / 2)-2]^{2}\right. \\
& +(J-3 / 2)(J+5 / 2)]\},
\end{aligned}
$$

and

$$
\left\langle{ }^{2} \Delta_{5 / 2}|\hat{H}|^{2} \Pi_{3 / 2}\right\rangle=-\bar{b}[(J-3 / 2)(J+5 / 2)]^{1 / 2},
$$

where all the band origins and rotational constants have their usual meanings. The $\bar{b}$ parameter arises from the orbitrotation operator $\left(-B \mathbf{J}_{ \pm} \mathbf{L}_{\overline{+}}\right)$ which has nonzero matrix element with selection rule $\Delta \Omega=\Delta \Lambda=\mp 1$.

Using the $2 \times 2$ matrix, we performed least squares fit to the measured term values of each vibrational level of both the ${ }^{2} \Pi_{3 / 2}$ and the ${ }^{2} \Delta_{5 / 2}$ states simultaneously. Since the $B, D$, and $\bar{b}$ parameters have similar forms of $J$ dependence, we experienced strong correlation among these parameters in the least squares fitting. In view of the fact that only relatively low $J$ transition lines $(J \leqslant 20.5)$ were measured, after some experimentation with the fitting program we eventually fixed the $D_{\Delta}$ and $D_{\Pi}$ parameters, respectively, at values of 0.2 $\times 10^{-6}$ and $0.1 \times 10^{-6} \mathrm{~cm}^{-1}$. Due to the difference in the vibrational constants for the two interacting states, as the vibrational quantum number increases the separation between the two interacting states also increases, the interaction between them decreases, and the $\bar{b}$ parameter was fixed at zero from the $v=4$ level onwards. The overall $\mathrm{rms}$ error of the fit was about $0.005 \mathrm{~cm}^{-1}$, which is slightly higher than the experimental limit of $0.002 \mathrm{~cm}^{-1}$. The results are listed in Table II. Equilibrium molecular constants for the $[14.0]^{2} \Delta_{5 / 2}$, $[15.1]^{2} \Delta_{5 / 2}$, and $[15.1]^{2} \Pi_{3 / 2}$ states of the isotopic molecules are listed in Table III.

We have also observed the $(1,0)(2,0)$, and $(5,0)$ bands for the $[15.1]^{2} \Delta_{5 / 2}-X^{2} \Pi_{3 / 2}$ transition of the ${ }^{60} \mathrm{Ni}{ }^{79} \mathrm{Br}$ and ${ }^{60} \mathrm{Ni}^{81} \mathrm{Br}$ isotopes, but due to the weakness of the $[15.1]^{2} \Pi_{3 / 2}-X^{2} \Pi_{3 / 2}$ transition the corresponding bands were not recorded. Individual transition bands were fit using our least squares program and the results are included in Table II. Both the two $(5,0)$ bands were fit properly with an overall rms error about $0.002 \mathrm{~cm}^{-1}$, but other bands were with larger rms errors between 0.012 and $0.020 \mathrm{~cm}^{-1}$ due to the fact that the perturbation from the $[15.1]^{2} \Pi_{3 / 2}$ was not accounted for.

\section{E. Isotopic relationships}

Molecular parameters of isotopic molecules are approximately related by different powers of the mass dependence $\rho=\left(\mu / \mu_{i}\right)$, where $\mu$ and $\mu_{i}$ are the reduced masses of ${ }^{58} \mathrm{Ni}{ }^{79} \mathrm{Br}$ and one of the isotopes, respectively. Since ${ }^{58} \mathrm{Ni}{ }^{79} \mathrm{Br}$ is the most abundant isotope, isotopic effects are calculated relative to it. Table III compares the observed and calculated equilibrium molecular parameters. The agreement of these values is good; the small discrepancy in the vibrational constants reflexes the slight inaccuracy in the band origins of the least squares fit due to the deperturbation procedures.

\section{F. Hyperfine structure in the $[15.1]^{2} \Delta_{5 / 2}-X^{2} \Pi_{3 / 2}$ band}

Figure 7 shows the band head region of the $(1,0)$ band of the $[15.1]^{2} \Delta_{5 / 2}-X^{2} \Pi_{3 / 2}$ transition. It is easily noticed that the $Q(2.5)$ line splits into two peaks and the $Q(3.5)$ and 
TABLE II. Molecular constants for the $[15.1]^{2} \Delta_{5 / 2}$ and $[15.1]^{2} \Pi_{3 / 2}$ states of $\mathrm{NiBr}\left(\mathrm{cm}^{-1}\right)$.

\begin{tabular}{|c|c|c|c|c|}
\hline Isotope & Parameter & {$[15.1]^{2} \Delta_{5 / 2}$} & {$[15.1]^{2} \Pi_{3 / 2}$} & $\bar{b}$ \\
\hline \multirow[t]{12}{*}{${ }^{58} \mathrm{Ni}^{79} \mathrm{Br}$} & $T_{0}$ & 15095.914 & 150.93 .446 & 0.121 \\
\hline & $B$ & 0.097871 & 0.094407 & \\
\hline & $T_{1}$ & 15390.902 & 15384.221 & 0.065 \\
\hline & $B$ & 0.097182 & 0.094106 & \\
\hline & $T_{2}$ & 15682.778 & 15671.042 & 0.059 \\
\hline & $B$ & 0.096635 & 0.093641 & \\
\hline & $T_{3}$ & 15973.630 & 15956.023 & 0.025 \\
\hline & $B$ & 0.095944 & 0.093140 & \\
\hline & $T_{4}$ & 16262.694 & 16239.131 & 0 \\
\hline & $B$ & 0.095269 & 0.092952 & \\
\hline & $T_{5}$ & 16549.821 & 16520.378 & 0 \\
\hline & $B$ & 0.094661 & 0.092633 & \\
\hline \multirow[t]{12}{*}{${ }^{58} \mathrm{Ni}{ }^{81} \mathrm{Br}$} & $T_{0}$ & 15095.999 & 15093.312 & 0.114 \\
\hline & $B$ & 0.096587 & 0.093189 & \\
\hline & $T_{1}$ & 15388.489 & 15382.791 & 0.101 \\
\hline & $B$ & 0.096178 & 0.093002 & \\
\hline & $T_{2}$ & 15679.455 & 15668.139 & 0.071 \\
\hline & $B$ & 0.095625 & 0.092632 & \\
\hline & $T_{3}$ & 15968.891 & 15951.657 & 0.030 \\
\hline & $B$ & 0.094945 & 0.0921185 & \\
\hline & $T_{4}$ & 16256.499 & 16233.323 & 0 \\
\hline & $B$ & 0.094281 & 0.091971 & \\
\hline & $T_{5}$ & 16542.229 & 16513.573 & 0 \\
\hline & $B$ & 0.093687 & 0.091706 & \\
\hline \multirow[t]{6}{*}{${ }^{60} \mathrm{Ni}^{79} \mathrm{Br}^{\mathrm{a}}$} & $T_{1}$ & 15386.884 & & \\
\hline & $B$ & 0.097044 & & \\
\hline & $T_{2}$ & 15676.747 & & \\
\hline & $B$ & 0.095278 & & \\
\hline & $T_{5}$ & 16535.780 & & \\
\hline & $B$ & 0.092806 & & \\
\hline \multirow[t]{6}{*}{${ }^{60} \mathrm{Ni}{ }^{81} \mathrm{Br}^{\mathrm{a}}$} & $T_{1}$ & 15385.112 & & \\
\hline & $B$ & 0.096290 & & \\
\hline & $T_{2}$ & 15673.354 & & \\
\hline & $B$ & 0.094152 & & \\
\hline & $T_{5}$ & 16528.087 & & \\
\hline & $B$ & 0.091867 & & \\
\hline
\end{tabular}

${ }_{\overline{\mathrm{a}}}^{\mathrm{S} \text { See text for explanation. }}$

$Q(4.5)$ have linewidth wider than the expected Doppler width. The structure and the larger linewidth arise from unresolved hyperfine structure, which is due to the interaction between the magnetic moment of the unpaired electron and the magnetic moment of an atom in the molecule. Since the ${ }^{58} \mathrm{Ni}$ and ${ }^{60} \mathrm{Ni}$ atoms have nuclear spin equal to zero, the observed hyperfine structure is expected to be caused by unpaired electrons interacting with the large magnetic moment of the bromine nucleus with nuclear spin $I=3 / 2$. The rapid decreases in linewidth as $J$ increases suggest that the coupling case is close to Hund's case $\left(\mathrm{a}_{\beta}\right)$ coupling scheme. ${ }^{40}$ Comparing linewidth of transition lines in other electronic systems studied, one can establish that the hyperfine structure occurs mainly in the excited $[15.1]^{2} \Delta_{5 / 2}$ state.

Magnetic hyperfine interactions in diatomic molecules were studied in detail by Frosch and Foley. ${ }^{41}$ The hyperfine Hamiltonian responsible for such interactions is
TABLE III. Equilibrium molecular constants for the $[14.0]^{2} \Delta_{5 / 2}$, $[15.1]^{2} \Delta_{5 / 2}$, and the $[15.1]^{2} \Pi_{3 / 2}$ states of ${ }^{58} \mathrm{Ni}{ }^{79} \mathrm{Br}$ and ${ }^{58} \mathrm{Ni}{ }^{81} \mathrm{Br}\left(\mathrm{cm}^{-1}\right)$. (Values given in parentheses are one standard error in last significant figures quoted.)

\begin{tabular}{|c|c|c|c|c|}
\hline \multirow[b]{2}{*}{ State } & \multirow[b]{2}{*}{ Parameter } & \multirow[b]{2}{*}{${ }^{58} \mathrm{Ni}{ }^{79} \mathrm{Br}$} & \multicolumn{2}{|c|}{${ }^{58} \mathrm{Ni}{ }^{81} \mathrm{Br}$} \\
\hline & & & Observed & Calculated $^{\mathrm{a}}$ \\
\hline \multirow[t]{6}{*}[14.0]{$^{2} \Delta_{5 / 2}$} & $T_{e}$ & 13842.3 & 13843.5 & $\ldots$ \\
\hline & $\omega_{e}$ & 288.3 & 286.1 & 286.8 \\
\hline & $\omega_{e} x_{e}$ & 1.57 & 1.42 & 1.55 \\
\hline & $B_{e}$ & 0.09546 & 0.09443 & 0.09446 \\
\hline & $\alpha_{e}$ & 0.00040 & 0.00032 & 0.00039 \\
\hline & $R_{e}(\AA)$ & 2.2990 & & \\
\hline \multirow[t]{6}{*}[15.1]{$^{2} \Delta_{5 / 2}$} & $T_{e}$ & 14 948.3(4) & $14949.0(2)$ & $\cdots$ \\
\hline & $\omega_{e}$ & 296.2(3) & 294.3(1) & 294.6 \\
\hline & $\omega_{e} x_{e}$ & $0.90(5)$ & $0.85(3)$ & 0.89 \\
\hline & $B_{e}$ & $0.09811(5)$ & $0.09701(7)$ & 0.09708 \\
\hline & $\alpha_{e}$ & $0.00061(1)$ & $0.00060(2)$ & 0.00060 \\
\hline & $R_{e}(\AA)$ & 2.2678 & & \\
\hline \multirow[t]{6}{*}[15.1]{$^{2} \Pi_{3 / 2}$} & $T_{e}$ & $14948.1(6)$ & $14948.8(8)$ & $\cdots$ \\
\hline & $\omega_{e}$ & $292.0(5)$ & $290.4(6)$ & 290.5 \\
\hline & $\omega_{e} x_{e}$ & $1.11(8)$ & 1.1(1) & 1.1 \\
\hline & $B_{e}$ & $0.09441(5)$ & $0.09333(7)$ & 0.09342 \\
\hline & $\alpha_{e}$ & $0.00031(1)$ & $0.00030(2)$ & 0.00030 \\
\hline & $R_{e}(\AA)$ & 2.3118 & & \\
\hline
\end{tabular}

$\overline{\bar{a} \text { Molecular constants were calculated using isotopic relationships and the }}$ values from ${ }^{58} \mathrm{Ni}^{79} \mathrm{Br}$.

$$
\hat{H}_{\mathrm{hfs}}=a I_{z} L_{z}+b \hat{I} \hat{S}+c I_{z} S_{z},
$$

where $a, b$, and $c$ parameters are the same as those defined by Frosch and Foley. ${ }^{41}$ The three terms in the hyperfine Hamiltonian are, respectively, the nuclear spin orbit, the Fermi contact, and the dipolar electron spin and nuclear spin interactions. For a $\Delta$ state with large spin-orbit interaction,

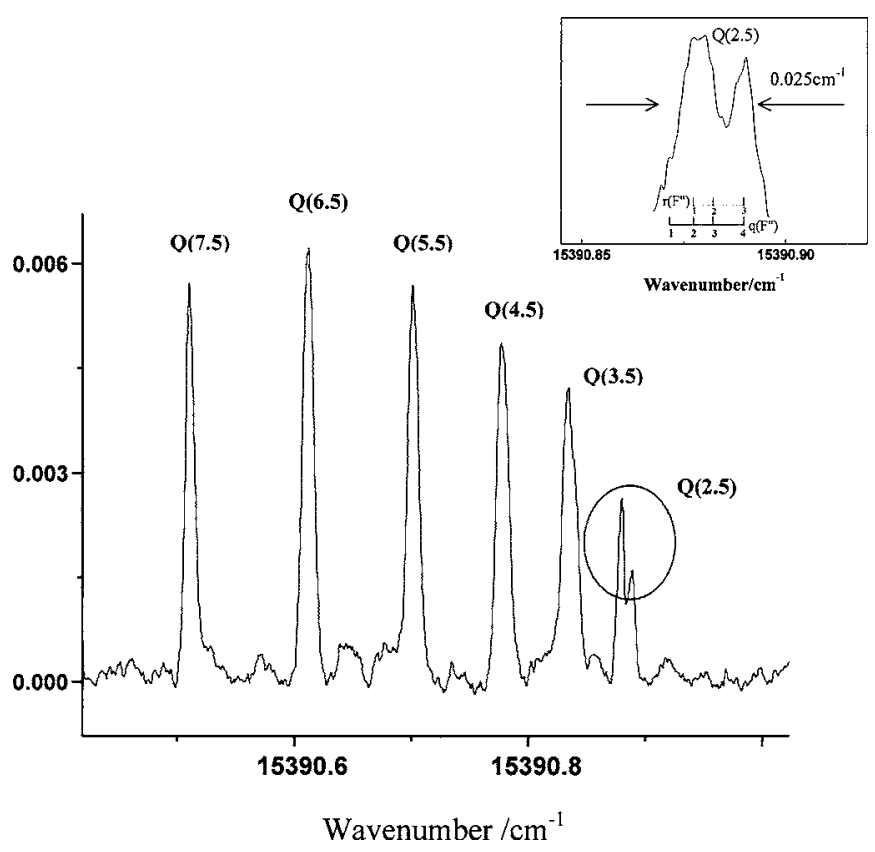

FIG. 7. The $Q$ branch of the $(1,0)$ band of the $[15.1]^{2} \Delta_{5 / 2}-X^{2} \Pi_{3 / 2}$ transition of ${ }^{58} \mathrm{Ni}{ }^{79} \mathrm{Br}$ with hyperfine structure. 


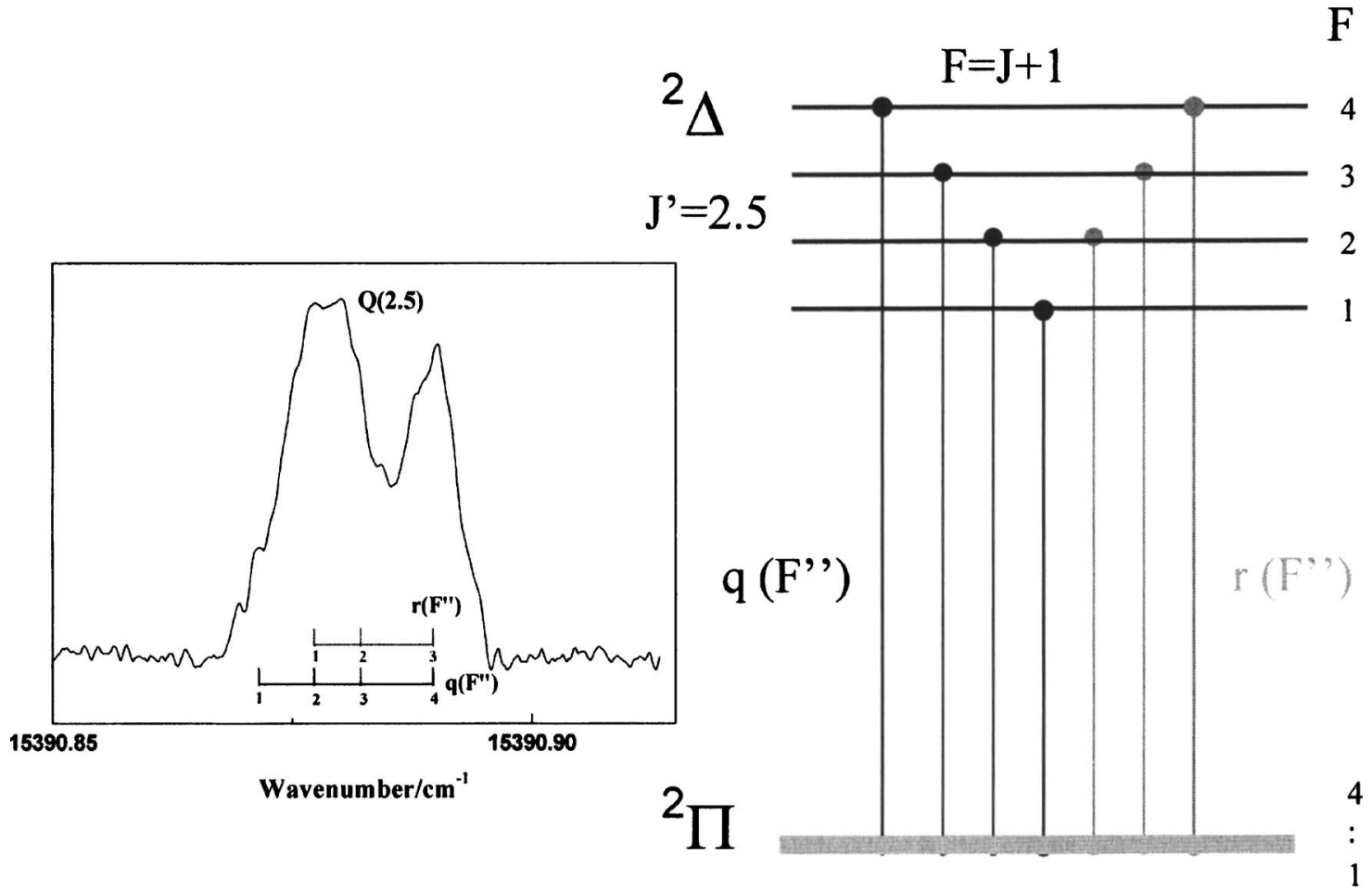

$\mathrm{J} "=2.5$

$$
\Delta \Lambda=+1 \quad \Delta \mathrm{F}=+1
$$

FIG. 8. Partially resolved $q\left(F^{\prime \prime}\right)$ and $r\left(F^{\prime \prime}\right)$ hyperfine structure of the $Q(2.5)$ line of the $(1,0)$ band of the $[15.1]^{2} \Delta_{5 / 2}-X^{2} \Pi_{3 / 2}$ transition of ${ }^{58} \mathrm{Ni}{ }^{79} \mathrm{Br}$.

the angular momentum coupling case is appropriately described by Hund's coupling case $\left(\mathrm{a}_{\beta}\right)$. In such a coupling case, the grand total quantum number $\mathbf{F}$ results from the coupling of the nuclear spin $\mathbf{I}$ with angular momentum $\mathbf{J}$, where $\mathbf{F}=\mathbf{I}+\mathbf{J}$. Hyperfine matrix elements of a $\Delta$ state in Hund's coupling case $\left(\mathrm{a}_{\beta}\right)$ can be found in the work of
Azuma et al. ${ }^{42}$ The matrix elements important for this analysis are

$$
\left\langle J \Omega I F\left|\hat{H}_{\mathrm{hfs}}\right| J \Omega I F\right\rangle=\frac{\Omega h[F(F-1)-I(I+1)-J(J+1)]}{2 J(J+1)},
$$

where $h=\Lambda a+(b+c) \sum$ for the ${ }^{2} \Delta_{5 / 2}$ substrate; with $\Lambda=2$ and $\Sigma=\frac{1}{2}$, this becomes $h=2 a+\frac{1}{2}(b+c)$. The observed rotationhyperfine line positions were fit in two steps. Initially, molecular parameters such as rotational constants and band origin of individual band were obtained in a band-by-band least squares fit and, subsequently, the hyperfine parameter was included for fitting the lines with hyperfine components. The hyperfine constant, $h_{5 / 2}$, for the $(1,0)$ band of the $[15.1]^{2} \Delta_{5 / 2}-X^{2} \Pi_{3 / 2}$ transition was determined to be $0.0039(8) \mathrm{cm}^{-1}$. With only partially resolved hyperfine structure as shown in Fig. 8, we have not been able to observe the vibrational dependence of the hyperfine constant. Theoretical calculations would be useful to find out the amount of con- tribution from the bromine atom from different molecular orbitals. Hyperfine constants in many studies were able to help evaluating such contributions; the $h_{5 / 2}$ constant determined in this work is a combination of $a, b$, and $c$ constants, which could not be interpreted fully unless other $h$ components are also determined.

\section{G. Discussion}

The molecular-orbital energy level diagram and some low-lying electronic states of $\mathrm{NiBr}$ have been discussed earlier. ${ }^{30}$ Many known electronic states of $\mathrm{NiBr}$ are expected to arise from the electronic configurations as follows: 


$$
\begin{aligned}
& (2 \sigma)^{2}(2 \pi)^{3}(1 \delta)^{4}, \quad X^{2} \Pi_{i} \\
& (2 \sigma)^{2}(2 \pi)^{4}(1 \delta)^{3}, \quad A^{2} \Delta_{i} \\
& (2 \sigma)^{1}(2 \pi)^{4}(1 \delta)^{4}, \quad{ }^{2} \Sigma^{+} \\
& (2 \sigma)^{2}(2 \pi)^{3}(1 \delta)^{3}(3 \sigma)^{1}, \quad(2)^{2} \Pi_{i},{ }^{4} \Pi_{i},(2){ }^{2} \Phi_{i},{ }^{4} \Phi_{i} \\
& (2 \sigma)^{2}(2 \pi)^{2}(1 \delta)^{4}(3 \sigma)^{1}, \quad{ }^{2} \Sigma^{+},{ }^{2} \Sigma^{-},{ }^{2} \Delta,{ }^{4} \Sigma^{-} \\
& (2 \sigma)^{2}(2 \pi)^{3}(1 \delta)^{3}(3 \pi)^{1}, \quad(2){ }^{2} \Sigma^{+},{ }^{4} \Sigma^{+},(2){ }^{2} \Sigma^{-},{ }^{4} \Sigma^{-},(4){ }^{2} \Delta_{i}
\end{aligned}
$$

The $[15.1]^{2} \Pi_{3 / 2}-X^{2} \Pi_{3 / 2}$ transition observed in this work is likely to be the promotion of an electron from the $1 \delta$ orbital to the slightly antibonding $3 \sigma$ orbital. The $[15.1]^{2} \Delta_{5 / 2}-X^{2} \Pi_{3 / 2}$ transition corresponds to the promotion of an electron from the $1 \delta$ orbital to the $3 \pi$ orbital. We also observed the $[15.1]^{2} \Pi_{3 / 2}-A^{2} \Delta_{5 / 2}$ and the $[15.1]^{2} \Delta_{5 / 2}-A^{2} \Delta_{5 / 2}$ transitions as indicated in our lowresolution spectrum, as depicted in Fig. 1. It should be noted that with the present association of the upper states to their corresponding electronic configurations, the $[15.1]^{2} \Pi_{3 / 2}-A^{2} \Delta_{5 / 2}$ and $[15.1]^{2} \Delta_{5 / 2}-A^{2} \Delta_{5 / 2}$ transitions could also be explained as the promotion of an electron, respectively, from $2 \pi$ orbital to $3 \sigma$ orbital and from $2 \pi$ orbital to $3 \pi$ orbital. All these transitions are with only one electron jump and are allowed transitions that are likely to be observable. The $[14.0]^{2} \Delta_{5 / 2}-X^{2} \Pi_{3 / 2}$ transition can be considered as the promotion of an electron from the $2 \pi$ orbital to the $3 \sigma$ orbital. This is consistent with the fact that there was no observed transition from the $A^{2} \Delta_{5 / 2}$ to the [14.0 $]^{2} \Delta_{5 / 2}$. This is because such transition requires the promotion of two electrons and it is highly unlikely that this transition would be strong enough to be observed.

Strictly speaking, there should be two parameters to fully describe the interaction between the $[15.1]^{2} \Delta$ and $[15.1]^{2} \Pi$ states; the off-diagonal spin-orbit $a_{+}$and the orbitrotation parameter $\bar{b}$. Due to large spin-orbit interaction in these electronic states, the spin-orbit components are separated by hundreds of wave numbers and resulted in case (c) coupling that only the $\Omega$ values are important. In our matrix for handling the interaction between the vibrational levels of the two electronic states, the $a_{+}$parameter was omitted; only the $\bar{b}$ parameter was used and determined from the least squares fit. In a diabatic model, the electronic part is often assumed to be independent of internuclear distance $R$. The nuclear and the electronic parts can be separated. The $\bar{b}$ parameter is a product of a vibrational and an electronic factor. $^{43}$ The interaction parameter becomes

$$
\begin{aligned}
\bar{b} & =\left\langle{ }^{2} \Delta\left(\pi^{3} \delta^{3} \pi^{1}\right), v\left|\left(\hbar^{2} / 2 \mu R^{2}\right) \Sigma_{i} l_{i}^{+}\right|^{2} \Pi\left(\pi^{3} \delta^{3} \sigma^{1}\right), v^{\prime}\right\rangle \\
& =\left\langle{ }^{2} \Delta\left(\pi^{3} \delta^{3} \pi^{1}\right)\left|\left(\hbar^{2} / 2 \mu R^{2}\right) \Sigma_{i} l_{i}^{+}\right|^{2} \Pi\left(\pi^{3} \delta^{3} \sigma^{1}\right)\right\rangle \cdot\left\langle v \mid v^{\prime}\right\rangle \\
& =b\left\langle v \mid v^{\prime}\right\rangle
\end{aligned}
$$

where $b$ is a parameter that depends on the electronic coordinates. The vibrational part is the overlap, $\left\langle v \mid v^{\prime}\right\rangle$, which can be calculated from the potential curves of the ${ }^{2} \Pi$ and ${ }^{2} \Delta$ states. The numerical values of the overlap elements evaluated using the Rydberg-Klein-Rees (RKR) potential energy curves ${ }^{44}$ constructed with molecular constants determined in this work are

$$
\begin{aligned}
& \left\langle v=0 \mid v^{\prime}=0\right\rangle=0.772, \quad\left\langle v=1 \mid v^{\prime}=1\right\rangle=0.446, \\
& \left\langle v=2 \mid v^{\prime}=2\right\rangle=0.254, \quad \text { and } \quad\left\langle v=3 \mid v^{\prime}=3\right\rangle=0.162
\end{aligned}
$$

It is expected that the vibrational dependence of $\bar{b}$ should be similar to that of $\left\langle v \mid v^{\prime}\right\rangle$ factor. The ratios, of $\left\langle v \mid v^{\prime}\right\rangle$ at $v=0$ to other $v^{\prime}$ levels are $0.57,0.34$, and 0.22 , which should be compared with the ratios of the $\bar{b}$ parameter at $v=0$ to other $v$ values which are $0.54,0.48$, and 0.20 . The agreement is excellent.

Zou and Liu ${ }^{5}$ recently performed comprehensive calculations to the four nickel monohalides. For $\mathrm{NiBr}$, they mapped our observed $[15.1]^{2} \Delta$ and $[15.1]^{2} \Pi$ states, respectively, to their calculated $(\mathrm{VI})_{5 / 2}$ and $(\mathrm{VIII})_{3 / 2}$ states. As pointed our earlier, our assignment of the $\Omega$ value to the excited states was based on the observation of the first lines in the transition, and for the $\Lambda$ value, the relative intensity of the $P, Q$, and $R$ branches was used. According to their results (Table IV of Ref. 5), the (VI) $)_{5 / 2}$ state is of (I) ${ }^{2} \Phi(65 \%)$ +(II) ${ }^{2} \Delta(34 \%)$ contributions which should have major character of a ${ }^{2} \Phi$ state; however, such theoretical assignment indicated that the relative intensities echo the dominant dipole transition: the contribution of (I) ${ }^{2} \Phi_{5 / 2}-X^{2} \Pi_{3 / 2}$ transition is likely to be smaller than (II) ${ }^{2} \Delta_{5 / 2}-X^{2} \Pi_{3 / 2}$ transition, even if the upper state is dominated by the (I) ${ }^{2} \Phi_{5 / 2}$ configuration. For the $(\mathrm{VIII})_{3 / 2}$ state has contributions from (II) ${ }^{2} \Delta(92 \%)$ which should have ${ }^{2} \Delta$ character. The two proposed theoretical states are $11 \mathrm{~cm}^{-1}$ apart, which resembles closely the observed states. The equilibrium bond length for the $[15.1]^{2} \Delta$ state is $2.268 \AA$ and the $[15.1]^{2} \Pi$ state is $2.312 \AA$, which is longer than the $(\mathrm{VI})_{5 / 2}$ state by $0.004 \AA$ and the $(\mathrm{VIII})_{3 / 2}$ state by $0.034 \AA$. As far as the ${ }^{2} \Phi$ state is concerned, we have recently observed a $[14.0]^{2} \Phi_{7 / 2}-X^{2} \Delta_{5 / 2}$ transition in NiI. ${ }^{35}$ Our recent work indicated that the $A^{2} \Delta_{5 / 2}$ state is only $37.5 \mathrm{~cm}^{-1}$ away from the ground state, which should be reasonably populated even under the supersonic jet condition, and the ${ }^{2} \Phi_{7 / 2}-A^{2} \Delta_{5 / 2}$ transition in NiBr should be observable.

\section{CONCLUSION}

We report here the rotational analysis and the perturbations found in the $v=0-3$ levels of the $[15.1]^{2} \Delta_{5 / 2}$ and the $[15.1]^{2} \Pi_{3 / 2}$ states. Using only the orbit-rotation interaction parameter $\bar{b}$, we have satisfactorily explained the observed perturbations. In addition, the hyperfine structure of the $(1,0)$ band of the $[15.1]^{2} \Delta_{5 / 2}-X^{2} \Pi_{3 / 2}$ system has been studied, and the hyperfine interaction was explained as arising from unpaired electrons interacting with the large magnetic moment of the bromine nucleus. 


\section{ACKNOWLEDGMENTS}

The Committee on Research and Conference Grants and the Research Grants Council of The Hong Kong Special Administrative Region, China, supported the work described here. The authors would like to thank P. M. Yeung and Dr. Jingjing Zheng for providing technical help in recording the spectra.

${ }^{1}$ C. W. Bauschlicber, Jr., S. P. Walch, and S. R. Langhoff, in Quantum Chemistry; The Challenge of Transition Metals and Chemistry, NATO Advanced Studies Institute, Series C: Mathematical and Physical Sciences, edited by A. Veillard (Reidel, Dordrecht, 1986).

${ }^{2}$ J. M. Brown and A. Carrington, Rotational Spectroscopy of Diatomic Molecules (Cambridge University Press, London, 2003).

${ }^{3}$ A. J. Merer, Annu. Rev. Phys. Chem. 40, 407 (1989).

${ }^{4}$ J. F. Harrison, Chem. Rev. (Washington, D.C.) 100, 679 (2000).

${ }^{5}$ W. Zou and W. Liu, J. Chem. Phys. 124, 154312 (2006).

${ }^{6}$ C. Dufour, P. Carette, and B. Pinchemel, J. Mol. Spectrosc. 148, 303 (1991)

${ }^{7}$ C. Dufour, I. Hikmet, and B. Pinchemel, J. Mol. Spectrosc. 158, 392 (1993).

${ }^{8}$ C. Dufour, I. Hikmet, and B. Pinchemel, J. Mol. Spectrosc. 165, 398 (1994).

${ }^{9}$ A. Bouddou, C. Dufour, and B. Pinchemel, J. Mol. Spectrosc. 168, 477 (1994).

${ }^{10}$ C. Dufour and B. Pinchemel, J. Mol. Spectrosc. 173, 70 (1995).

${ }^{11}$ C. Focsa, C. Dufour, and B. Pinchemel, J. Mol. Spectrosc. 182, 65 (1997).

${ }^{12}$ Y. Chen, J. Jin, C. Hu, X. Yang, X. Ma, and C. Chen, J. Mol. Spectrosc. 203, 37 (2000)

${ }^{13}$ J. Jin, Y. Chen, X. Yang, Q. Ran, and C. Chen, J. Mol. Spectrosc. 208, 18 (2001)

${ }^{14}$ J. Jin, Q. Ran, X. Yang, Y. Chen, and C. Chen, J. Phys. Chem. A 105, 11177 (2001).

${ }^{15}$ M. Tanimoto, T. Sakamaki, and T. Okabayashi, J. Mol. Spectrosc. 207, 66 (2001).

${ }^{16}$ Y. Krouti, T. Hirao, C. Dufour, A. Boulezhar, B. Pinchemel, and P. F. Bernath, J. Mol. Spectrosc. 214, 152 (2002).

${ }^{17}$ B. Pinchemel, T. Hirao, and P. F. Bernath, J. Mol. Spectrosc. 215, 262 (2002).

${ }^{18}$ T. Hirao, C. Dufour, B. Pinchemel, and P. F. Bernath, J. Mol. Spectrosc. 202, 53 (2000).

${ }^{19}$ A. Poclet, Y. Krouti, T. Hirao, B. Pinchemel, and P. F. Bernath, J. Mol.
Spectrosc. 204, 125 (2000).

${ }^{20}$ Y. Krouti, A. Poclet, T. Hirao, B. Pinchemel, and P. F. Bernath, J. Mol. Spectrosc. 210, 41 (2001)

${ }^{21}$ E. Yamazaki, T. Okabayashi, and M. Tanimoto, Astrophys. J. Lett. 511, L199 (2001).

${ }^{22}$ L. C. O’Brien, K. M. Homann, T. L. Kellerman, and J. J. O’Brien, J. Mol. Spectrosc. 210, 41 (2001).

${ }^{23}$ J. J. O'Brien, J. S. Miller, and L. C. O’Brien, J. Mol. Spectrosc. 211, 93 (2002).

${ }^{24}$ S. Tumturk, L. C. O’Brien, and J. J. O’Brien, J. Mol. Spectrosc. 255, 225 (2004).

${ }^{25}$ C. A. Rice and L. C. O’Brien, J. Mol. Spectrosc. 221, 131 (2003).

${ }^{26}$ A. B. Darji and N. R. Shah, Curr. Sci. 48, 349 (1979).

${ }^{27}$ R. Gopal and M. M. Joshi, Curr. Sci. 50, 1061 (1981).

${ }^{28}$ R. Gopal and M. M. Joshi, Indian J. Phys., B 59, 309 (1985).

${ }^{29}$ S. P. Reedy, N. Narayana, and P. T. Rao, Opt. Pura Apl. 20, 69 (1987).

${ }^{30}$ J. W. H. Leung, X. Wang, and A. S.-C. Cheung, J. Chem. Phys. 117, 3694 (2002)

${ }^{31}$ E. Yamazaki, T. Okabayashi, and M. Taninmoto, J. Chem. Phys. 121, 162 (2004).

${ }^{32}$ W. S. Tam, J. W.-H. Leung, S. Hu, and A. S.-C. Cheung, J. Chem. Phys. 119, 12245 (2003)

${ }^{33}$ W. S. Tam, J. Ye, and A. S.-C. Cheung, J. Chem. Phys. 121, 430 (2004).

${ }^{34}$ Q. Ram, W. S. Tam, C. Ma, and A. S.-C. Cheung, J. Mol. Spectrosc. 198, 175 (1999).

${ }^{35}$ A. S.-C. Cheung, W. S. Tam, J. Ye, and J. W.-H. Leung, (unpublished).

${ }^{36}$ A. S.-C. Cheung, Chin. J. Chem. Phys. 17, 299 (2004).

${ }^{37}$ G. Herzberg, Spectra of Diatomic Molecules (Van Nostrand, New York, 1950).

${ }^{38}$ See EPAPS Document No. E-JCPSA6-125-009647 for the observed line positions of the $[15.1]^{2} \Delta_{5 / 2}-X^{2} \Pi_{3 / 2}, \quad[15.1]^{2} \Pi_{3 / 2}-X^{2} \Pi_{3 / 2}$, and $[14.3]^{2} \Delta_{5 / 2}-X^{2} \Pi_{3 / 2}$ transitions. This document can be reached via a direct link in the online article's HTML reference section or via the EPAPS homepage (http://www.aip.org/pubservs/epaps.html).

${ }^{39}$ S. F. Rice, W. J. Child, and R. W. Field, J. Mol. Spectrosc. 133, 22 (1989).

${ }^{40}$ T. M. Dunn, in Molecular Spectroscopy: Modern Research, edited by K. N. Rao (Academic, New York, 1972), Vol. III, Chap. 4.4.

${ }^{41}$ R. A. Frosch and H. M. Foley, Phys. Rev. 88, 1337 (1952).

${ }^{42}$ Y. Azuma, J. A. Barry, M. P. J. Lyne, A. J. Merer, J. O. Schroder, and J. L. Femenias, J. Chem. Phys. 91, 1 (1989).

${ }^{43} \mathrm{H}$. Lefebvre-Brion and R. W. Field, The Spectra and Dynamics of Diatomic Molecules (Elsevier, New York, 2004).

${ }^{44}$ R. J. LeRoy, University of Waterloo Chemical Physics Research Report No. CP-657R, 2004 